\title{
Single Sink Repositioning Technique in Wireless Sensor Networks for Increasing Throughput and Decreasing Delay
}

\author{
Asrat Sime Bedada ${ }^{1}$ Mebratu Fana Bedasa ${ }^{2}$ Wesenu Bekele Mulatu ${ }^{3}$ \\ 1.Department of Computer Science, College of Computing, Madda Walab University, Bale Robe, Ethiopia \\ 2.Department of Computer Science, College of Computing, Madda Walabu University, Bale Robe, Ethiopia \\ 3.Department of Information Systems, College of Computing, Madda Walabu University, Bale Robe, Ethiopia
}

\begin{abstract}
Wireless sensor network (WSN) refers to a group of spatially spread and enthusiastic sensors for monitoring and recording the physical conditions of the environment and organizing the collected data at a central location. WSN becomes an energetic subject with the rapid development that is vulnerable to a varied high-quality of attacks due to deployment in the hostile environment. In WSN, throughput is defined as the amount of data transferred from one sensor node to another in a specified amount of time. Delay It refers to the total time taken for a single packet to be transmitted across a network from source to destination. The basic idea of sink relocation is to shorten the distance between sink node and sensor node. So, that significant power savings can be achieved. The problem of distant node energy consumption of wireless sensor node was solved by sink repositioning technique which has capability to move and communicate with all sensor node inside the region. The sink node and sensor node are randomly deployed within the geographic extent of the entire network. In order to test for the impact of repositioning the total power transmission of the sensors for the previous and next sink positions is evaluated and compared. This is result in increasing life time of sensor network by putting sink node at optimum location to decrease the distant node, increases throughput and decrease delay with sink node and analyze the difference. The main aim of the study was to increase throughput and decrease end to end delay. For this study we have used NS2.35 environment for simulation. Our simulation results show that repositioning the sink achieves significant change on throughput and delay when compared to the static sink approach that have been presented by using network animator(NAM) and Xgraph.
\end{abstract}

Keywords: WSN, Sink node, sensor node, throughput, delay and sink reposition.

DOI: $10.7176 /$ CEIS/11-5-01

Publication date:September $30^{\text {th }} 2020$

\section{Introduction}

WSN refers to a group of spatially dispersed and dedicated sensors for monitoring and recording the physical conditions of the environment and organizing the collected data at a central location. WSNs measure environmental conditions like temperature, sound, pollution levels, humidity, wind, and so on. Recent years have observed a high interest in the use of WSN in abundant applications such as forest monitoring, disaster management, space exploration, factory automation, secure installation, border protection, and battlefield surveillance [1][2]. WSN becomes an energetic subject with the rapid development that is vulnerable to a varied high-quality of attacks due to deployment in the hostile environment. Such networks have been understood due to recent advances in micro electromechanical systems and are expected to be widely used for applications such as environment monitoring, home security, and earthquake warning. In addition to the ability to enquiry its surroundings, each sensor has an onboard radio to be used for sending the collected data to a sink Base station (BS) either directly or over a multi-hop path. Sensor node is a small device, which is used to gather data from its neighboring area, perform simple computations, and communicate with other sensors or with BS [3]. Because of this property, Attribute of sensor networks, is the cluster of sensor nodes, to generate high class information about the sensing surroundings. These nodes are self-organized. They are competent of wireless communication. It is forced in circumstances of memory; dimension, energy, processing power and sense environmental data [4]. Thus; a WSN has sensing component as well as the capabilities of, on-board processing, storage and communication. By these improvements, a sensor is prone for data collection correlation, in network examination and combination of other sensor nodes data and its own sensor data also. While several sensors nodes supervise the large physical environment, they structure a WSN. There are basically two components in the infrastructure of a wireless sensor network: sink nodes and sensor nodes. Sink nodes are considered as a BS in the network that wirelessly receive and collect data packages generated from all the sensor nodes in the network and provide them to users. A sink node or BS acts like an interface between users and the network. From the BS, users can access the data, possibly through internet, for further processing of the data and to extract useful information. Depending on the network size and network topology, there could be one or multiple sink nodes and the sink nodes can either be stationary at one position or touring in the network area. The sink node with BS functionality is usually supplied with large energy reserve and large computational power as it works as a pivot in the WSN system [5][13].

In WSN, throughput is defined as the amount of data transferred from one sensor node to another in a specified 
amount of time. Typically, throughput is usually measured in bits per second (bits/s or bps), and sometimes in data packets per second or data packets per time slot. In this article, the throughput is measured in data packets bits per second (bits/s or bps). Maximum throughput is the largest amount of data volume that can be generated by the entire network. Maximum throughput routing is the routing path which could lead to maximum throughput for a sensor. Delay plays a very important role in WSN. It refers to the total time taken for a single packet to be transmitted across a network from source to destination. Delay can take a long time for a packet to be delivered across intervening networks. In reliable protocols where a receiver acknowledges delivery of each chunk of data, it is possible to measure this as round-trip time. Due to congestion problem some time traffic will be occurs in the network. So, delay will be increases and reliability of data transmission also decreases. Delay factor directly effect on network performance [9][10].

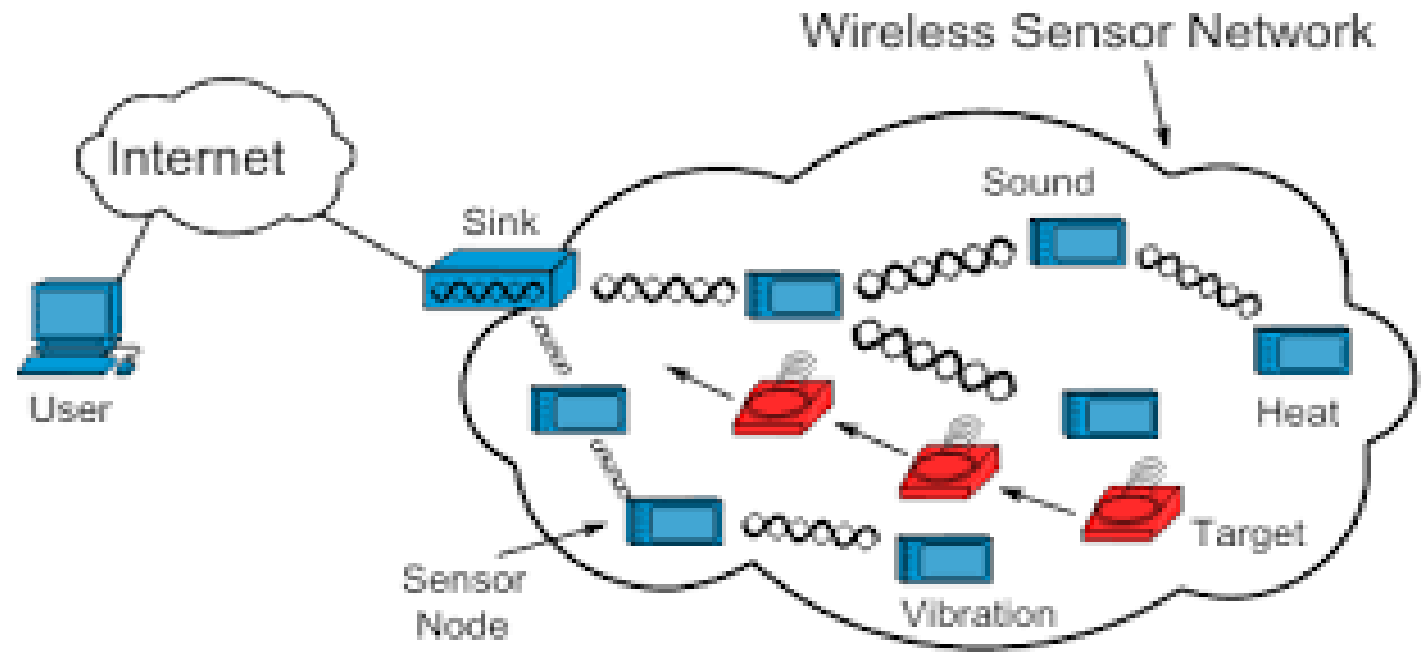

Figure 1. WSN environments

\section{Problem Identification}

Sensors are usually battery operated and have a limited transmission range and on-board processing capacity like memory, processing, energy and power. Such constraints have motivated lots of research on effective management strategies of WSN's that trade off resources, data fidelity, delay, throughput and coverage so that the network can stay functional for the longest duration. energy efficiency has become a primary issue in WSN. The sensor networks are powered by battery and thus they turn out to be dead after a particular interval. Hence, enhancing the data dissipation in energy efficient manner remains to be more challenging for increasing the life span of sensor devices. It has been already proved that the single sink repositioning technique method to enhance the life span of WSNs. The interest in optimizing the transmission energy tends to increase the levels of packet relaying and thus makes queuing delay an issue especially for real time traffic. The wireless sensor node can only be equipped with a limited power source. Sensor lifetime shows a strong dependency on battery lifetime.

Energy efficient data routing in WSN generally pursue sink relocation for minimizing the total transmission power which is generally proportional to the distance squared or even higher in order for environment reach with obstacles and interference sources. The basic idea of sink relocation is to shorten the distance so that significant power savings can be achieved. But, in case of WSN all the sensors will forward their data to the sink. So, the sensors which are nearer to the sink have less delay and high throughput because as distance decrease delay between sensor node and sink nodes are decreases and throughput increases. Now the sink will have to rely on the other sensors which are quite far off. This will result in increases delay and decreases throughput. Thus, in case of sink communicating sensors have to communicate a lot of traffic for the other sensors and this gives rise to unbalanced throughput and end to end delay. However, to provide solution over this problem some methods were proposed wherein they tried to solve this problem.[6][7] [4][13]. The main objective of this study was on the increasing of throughput and decreasing end to end delay by using sink relocation method.

\section{Related Work}

Some of the related works conducted by foreign and local researchers have been reviewed as follows.

Asrat Sime et al. [13] they presented approach considers relocation of the sink node by checking the location of the nodes that are distant-hop away from the sink and their distance from the sink node. Long distance nodes consume more energy than other node. Energy consumption of node is directly proportional with the distance. In this paper they have investigated the performance advantage of repositioning the sink node of WSN. Repositioning of the sink increases the average lifetime of the nodes by decreasing the average energy consumed per packet. It 
achieves significant amount of energy savings as compared to the base line approach. In this study, the authors were only used energy to increase the life time of battery. But, they were not considered other parameters such as throughput and delay.

Tapan Kumar et al. [14] the proposed work performs network lifetime optimization through energy balancing in a multiple sink single hop WSN. The network restructuring balances the energy amongst the sinks, thereby increasing the network lifetime. In the proposed algorithm they have considered the network lifetime with respect to the maximum and average energy consumption. The more the energy consumed, the less the network lifetime. The proposed restructuring algorithm reduces the maximum nodes connected to a sink. As a result, the total energy consumed by the sink connected to the maximum nodes also decreases further resulting in increasing the total network lifetime.

Wint Yi Poe et al. [15] they had proposed local search technique for sink placement in WSNs that tries to minimize the maximum worst-case delay and extend the lifetime of a WSN simultaneously. Since it is not feasible for a sink to use global information, which especially applies to large-scale WSNs, they introduce a self-organized sink placement (SOSP) strategy. The main goal of this study was to provide a better sink placement strategy with a lower communication overhead. Avoiding the costly design of using nodes' location information, sink sets up its own group by communicating to its n-hop distance neighbors. But, they did not study the difference throughput and end to end delay before and after sink reposition.

From the review made, most of the papers were done on optimizing energy consumption by repositioning sink node in the transmission region. When the sink node repositions, the energy consumption of the longest node was optimized and the life time of node was increased, but they did not evaluate the difference on throughput and end to end delay during sink move to optimum position. When the sink moves to optimum position the energy consumption of single node decrease as distance between sink node and sensor node decreases. During this, the throughput is increase and end to end delay is decrease. The key objective of this study was to evaluate the difference between throughput and delay before and after sink reposition.

\section{The Proposed Methodology}

The problem of energy consumption of WSN nodes was solved by sink repositioning technique that used mobile sink nodes which has capability to move inside region and communicate with all sensor nodes inside region. The sink node and sensor nodes are randomly deployed within the geographic extent of the entire network. If the sink node is in optimum location in geographic extent, all sensor node directly communicates with sink node without repositioning sink. If the sink node is not in optimum location the distance of each node from sink node is calculated, and then the optimal location for the sink is also calculated. Then the sink node repositions (move) to optimum location inside a region. Because, the highest distance node from sink consume more energy to transmit packet than other nearest node and these nodes deplete its energy before all other node which lead to energy hole problem. In order to test for the impact of repositioning, the total power transmission of the sensors for the previous and next sink positions is evaluated and compared. This is result in increasing life time of sensor network by putting sink node at optimum location to decrease the distant node and decrease energy consumption to communicate with sink node. After energy consumption of each node is optimized, we are going to calculate throughput and delay before sink repositioning and after sink repositioning to analyze the differences. The main aim of the study is to increase throughput and decrease delay. When the distance between sink node and sensor node decreases the total delay also decreases and throughput increases and when distance increases the delay also increases and throughput decreases which affect the quality of communication over WSN. Figure 2 shows flowchart to calculate throughput and delay. 


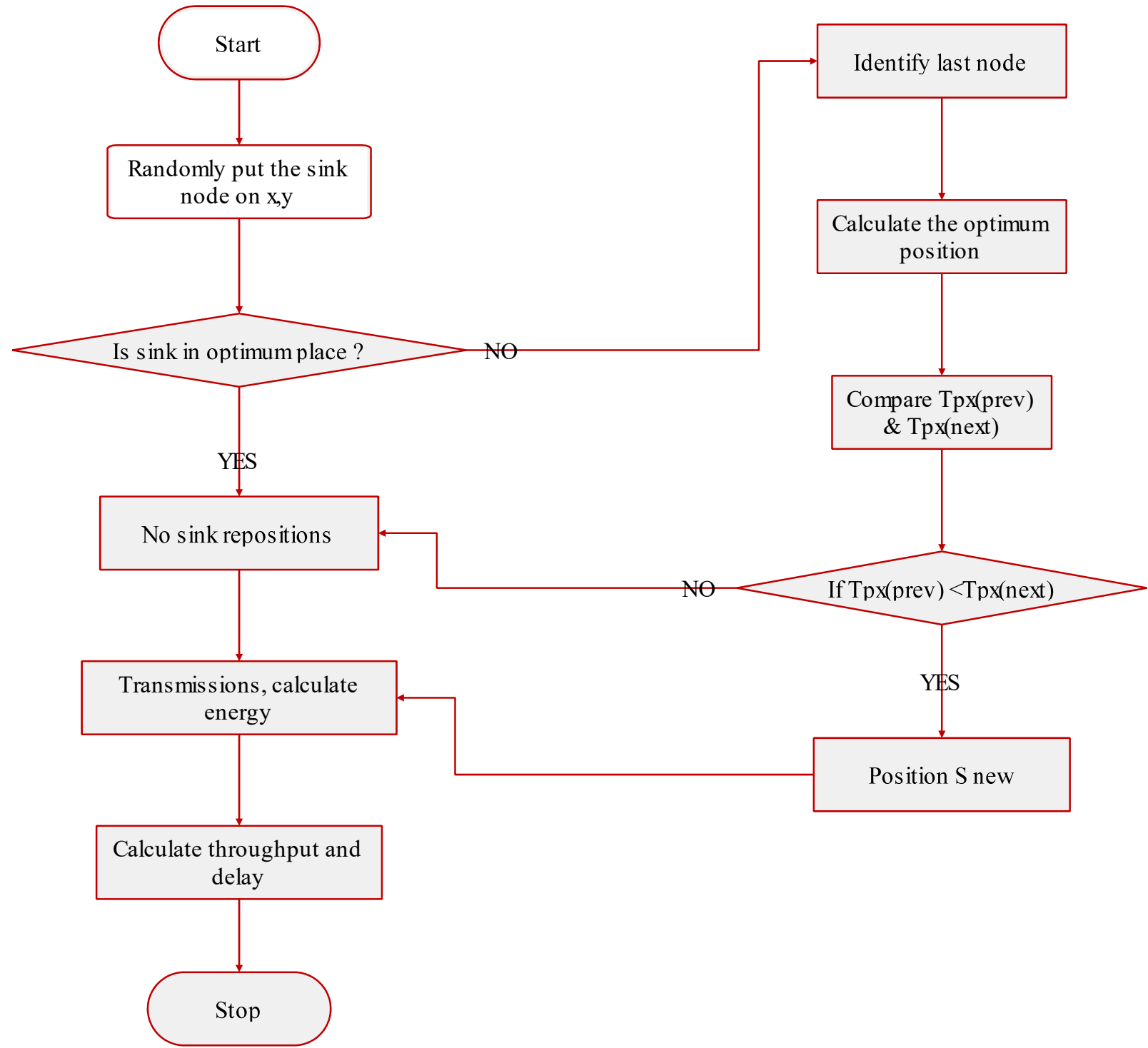

Figure 2. Flow chart of calculating throughput and delay over sink relocation

\subsection{Algorithm /Pseudocode}

1. All sensor nodes are randomly deployed in the transmission boundaries of $X, Y$ Position.

2. The sensor nodes after random deployment are fixed position.

3. Select sink node $S$ from wireless nodes in the transmission boundaries of $X, Y$ Position.

4. Put Sink node $S$ randomly in the $(X, Y)$ position.

5. The sinks have more power than the sensing nodes. The sinks have additional computational capacity as well.

6. Calculate the distance D between each node and sink node using Euclidean distance formula [11]. $D(X, Y)=\sqrt{(\mathrm{x} 1-\mathrm{y} 1)^{2}+(\mathrm{x} 2-\mathrm{y} 2)^{2}}$ $-(1)$

7. Calculate Energy consumption Etx of each node to transmit and receive packet based on distance from sink node by using energy consumption formula [12].

ETx $(\mathrm{k}, \mathrm{d})=$ Eelec $\times \mathrm{k}+$ Eamp $\times \mathrm{k} \times \mathrm{d}^{\mathrm{n}}$

8. Calculate average position for Sink node in the transmission boundaries $(X, Y)$.

$\operatorname{avg} X=(\operatorname{sumx}(D) /$ number of node, $\operatorname{avg} Y=(\operatorname{sumy}(D) /$ number of nodes New position for Sink node is $(\operatorname{avg} X, \operatorname{avg} Y)$

9. Repositioning the sink node to new calculated position for sink node (avg X, avgY) in the transmission boundaries.

10. Then repeat step 6 and step 7 respectively.

11. Compare the distance and energy consumption of each node before and after sink repositioning. 


\section{Calculate throughput. \\ 13. Calculate delay.}

\section{System Model and Parameters}

The Network Simulator (NS-2.35) is used to simulate the proposed architecture. In the simulation, the wireless node and sink nodes in a 1400-meter x 1400-meter region for 100 seconds of simulation time. The proposed single sink relocation is mobile in side transmission region until it gets optimum location. After sink reaches its optimum location sink node stay new position permanently and receive data from other sensor nodes. The performance is evaluated mainly distance between each node and sink node, examine the change of throughput and delay before and after sink reposition. The objective of study was to compare throughput and delay before and after sink reposition.

\subsection{Parameters used}

Table 1. Parameters used

\begin{tabular}{|c|c|}
\hline Parameters & Value \\
\hline Area size & $1400^{*} 1400 \mathrm{~m}^{2}$ \\
\hline Traffic source & CBR \\
\hline MAC & IEEE 802.15 .4 \\
\hline Routing protocol & AODV \\
\hline Simulation time & $100 \mathrm{~s}$ \\
\hline Idle power & $0.035 \mathrm{~J}$ \\
\hline Transmission power & 0.660 \\
\hline Receive power & 0.395 \\
\hline Initial energy & $0.01 \mathrm{~J}$ \\
\hline Number of nodes & 49 \\
\hline Size of data packet & 8 bits \\
\hline
\end{tabular}

\section{Result and Discussion}

The simulations are carried out using NS-2.35. we get a NAM file which should be opened in.NAM console to see the visualization of our WSN , the graphs were plotted using XGraph and the outputs are generated using AWK file.

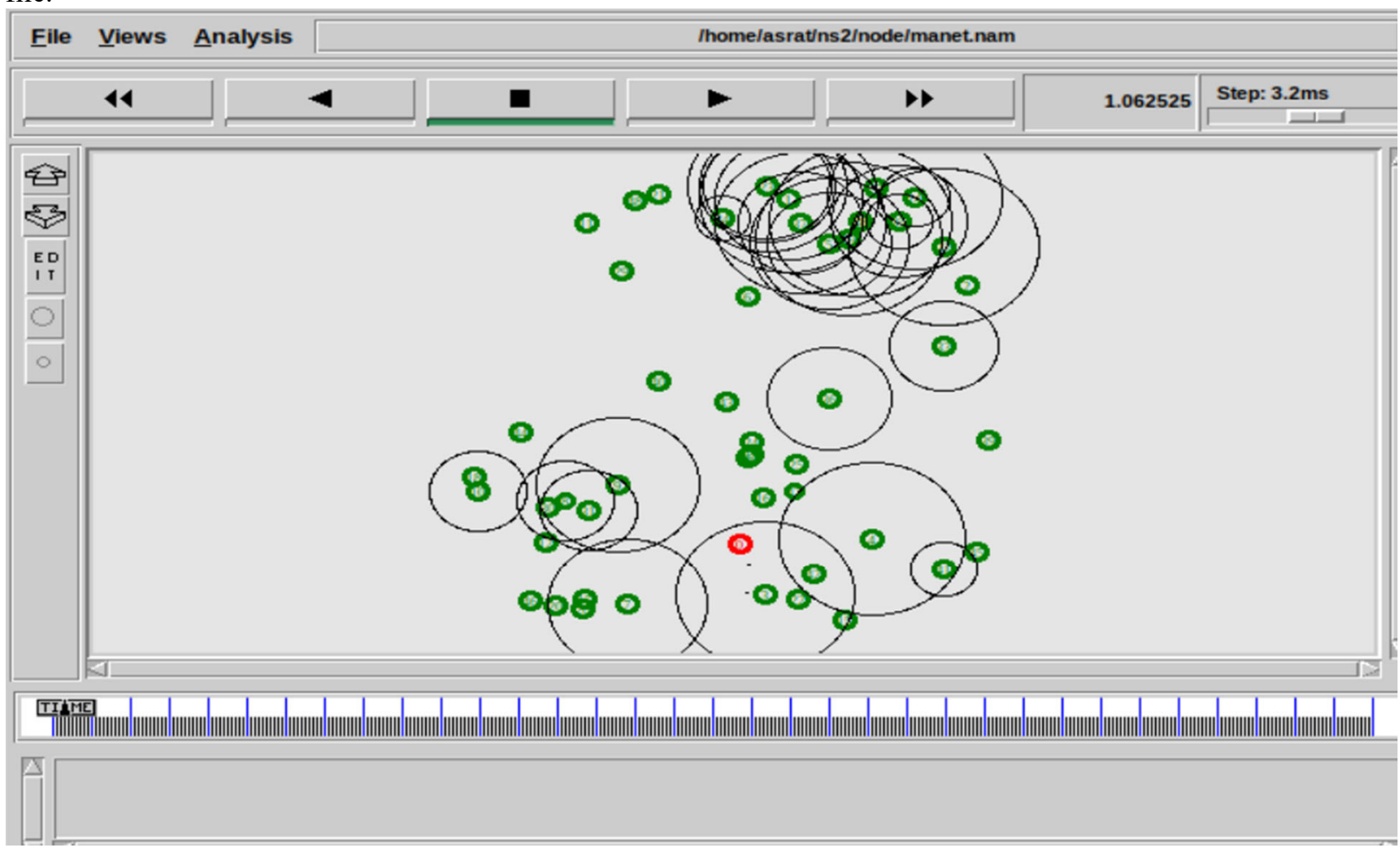

Figure3. Output before sink repositioning

Figure 3 displays that node sink node colored by red color is selected as sink node before reposition. As we see from figure 4 some nodes have long distance from sink node while some nodes are near to the sink. So, the 
distant node has less throughput and maximum delay. To overcome these problems, sink node start reposition to optimum location in the region as we have seen from figure 4 below.

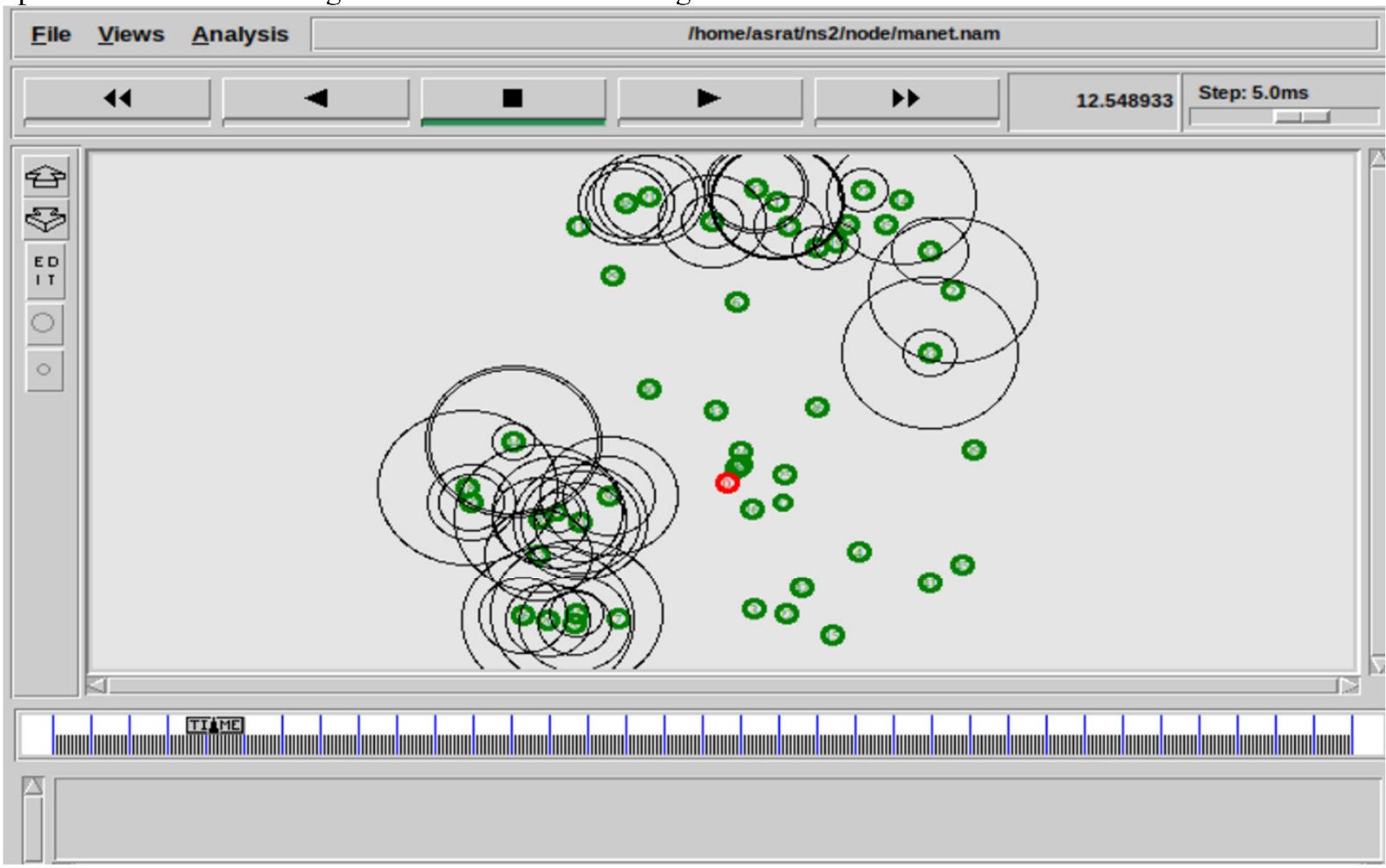

Figure 4. Output sink node start moving to optimum position

Figure 4 shows that during sink node starting drive to optimal location. During sink node drive to optimal location the highest distance node adjusts its transmission power as well as throughput and delay as remoteness decreases and energy consumption also decreased. As we have seen from figure 5 below, sink node reach optimum location stay lastingly after it reaches its optimal location

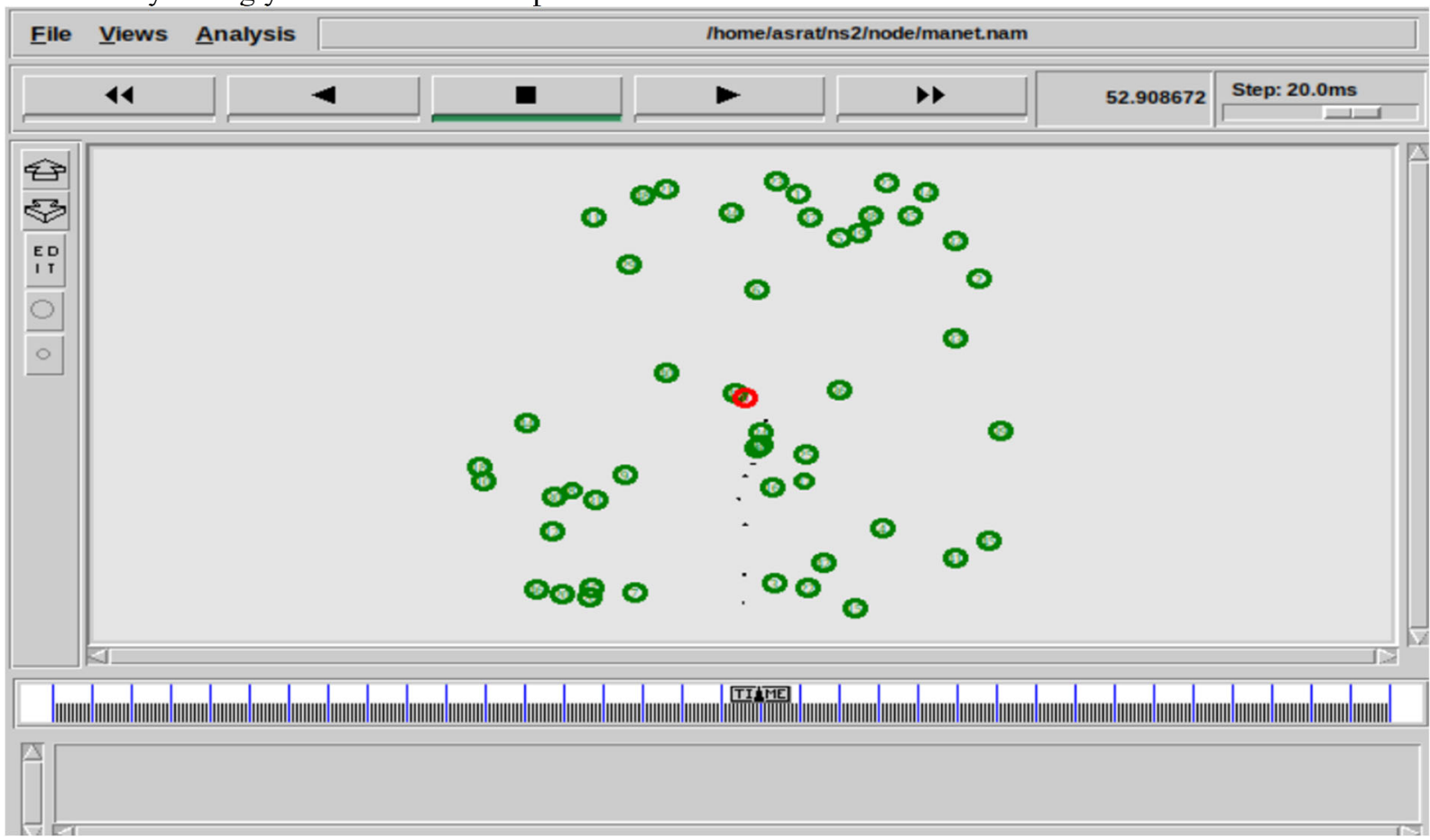

Figure 5. Output after sink repositioning to optimum location

Figure 5 shows that after sink node reach optimal location and receive packet from nodes. Sink node stay lastingly after it reaches its optimal location and remain its communication with nodes. Sink repositioning helps to avoids consuming too much battery energy for a specific group of sensor nodes as well as too small consuming 
battery energy for a specific group of sensor nodes as well. This can result in increasing of throughput and decreasing of end to end delay.

\section{Xgraph Output}

\subsection{Test case 1:}

This test case is for AODV protocol on wireless sensor node that shows the difference on throughput and delay before and after sink node reposition to central (optimum) position on the XY plane. The xgraph indicates that throughput and delay which are traced by the trace file

\subsubsection{Output of Xgraph Before Sink Node Repositioning}

\section{( $)$ (ㅁ) xgraph}

Close Hdcpy About Anim Replot Deriv

X Gaph

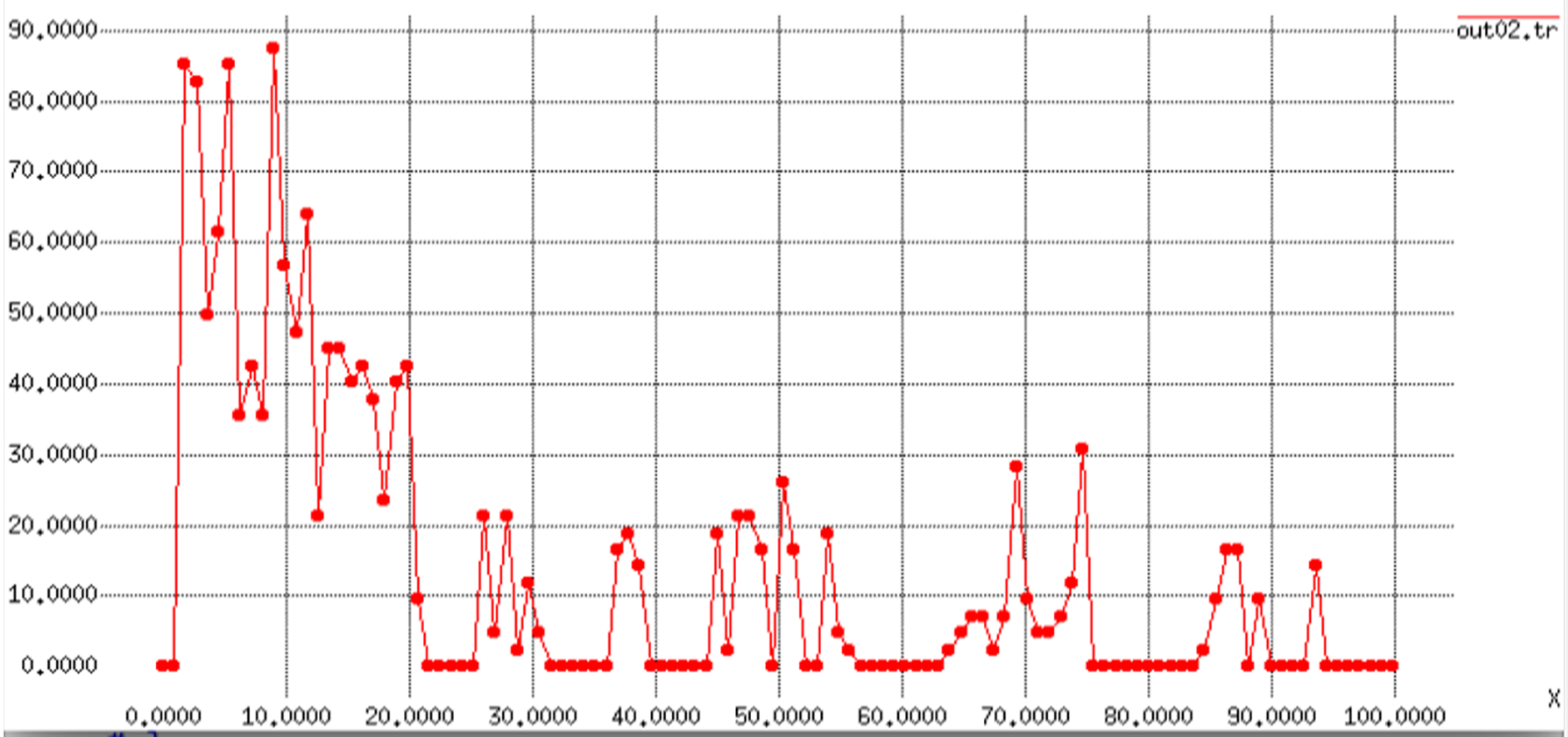

Figure 6. A screenshot depicting throughput in test case 1

Figure 6 shows the throughput values before sink repositioning. Sink node randomly placed on XY plane and directly communicate with another sensor node from fixed place. As we have seen from the graph throughput was decreases as time increases.

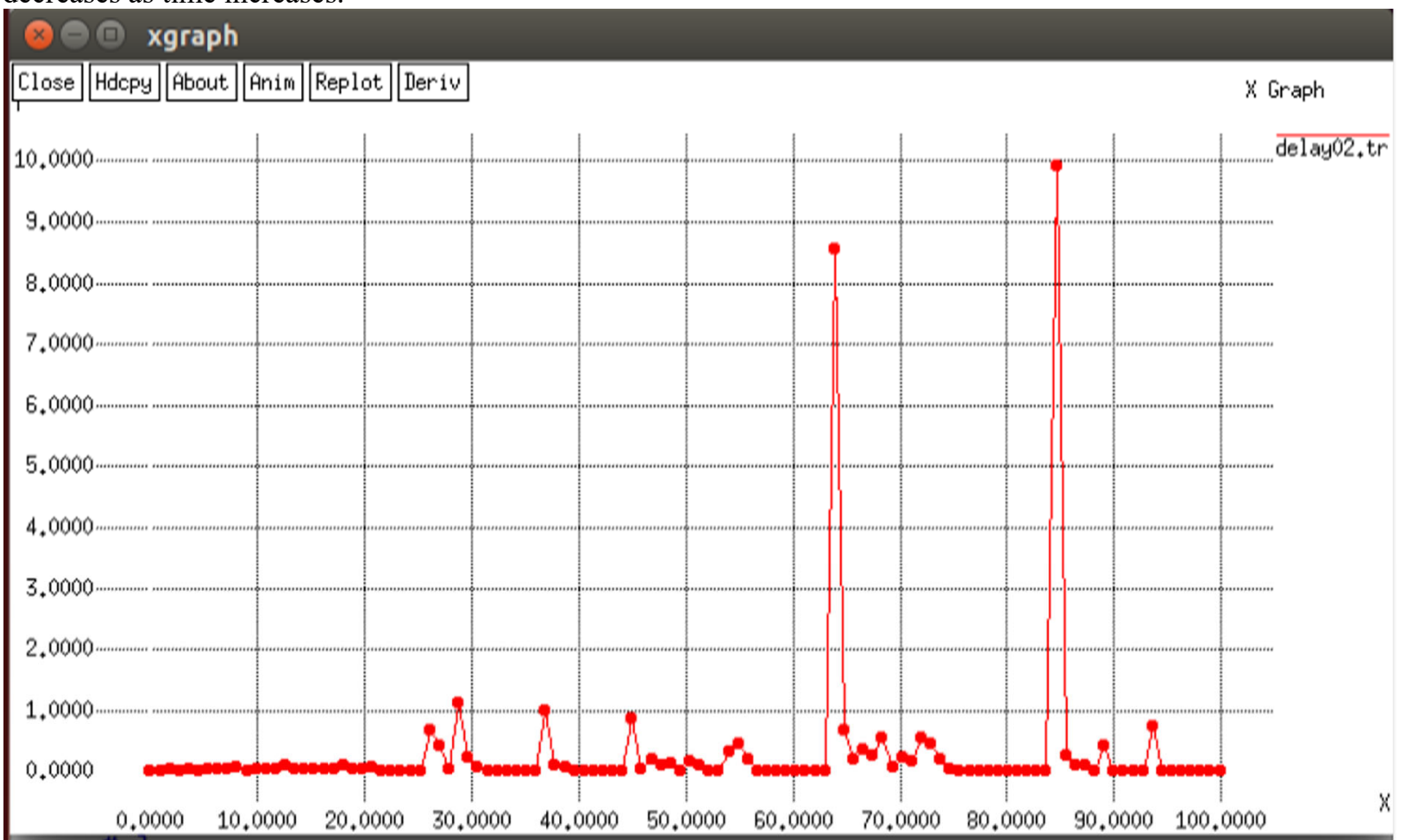

Figure 7. A screenshot depicting delay in test case 1 
Figure 7 shows the delay in the test case when sink node is at fixed location or before sink reposition. The result of AODV protocols are clearly show that delay is less at start time and increases as time increases.

Test case 1 is tested for AODV output figure 8 number of sent packet, number of received packet, average end-to end delay, number of dropped packet and throughput before sink reposition.

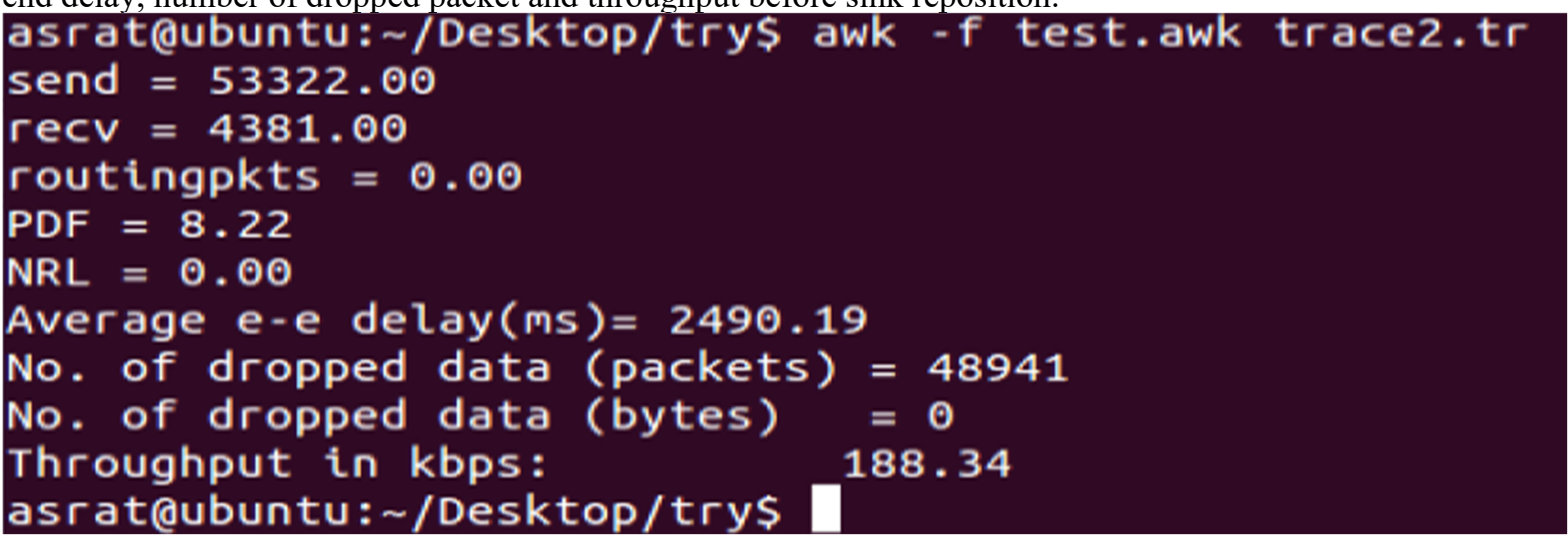

Figure 8 . shows the screenshot of packet information in test case 1 .

Average end-to-end delay $=2490.19 \mathrm{~ms}$

Throughput $=188.34 \mathrm{kbps}$

Figure 8 shows the throughput is less and average end to end delay is high which affect the quality of communication in WSN.

\subsection{Test case 2:}

7.2.1 Output of Xgraph After Sink Node Repositioning

This test case to show the difference output with test case 1 . Since our sink nodes is randomly placed on XY plane and move to optimum location. So, the throughput and delays are different from test case 1. Figure 8 and 9 shows the screenshot of throughput in this case.

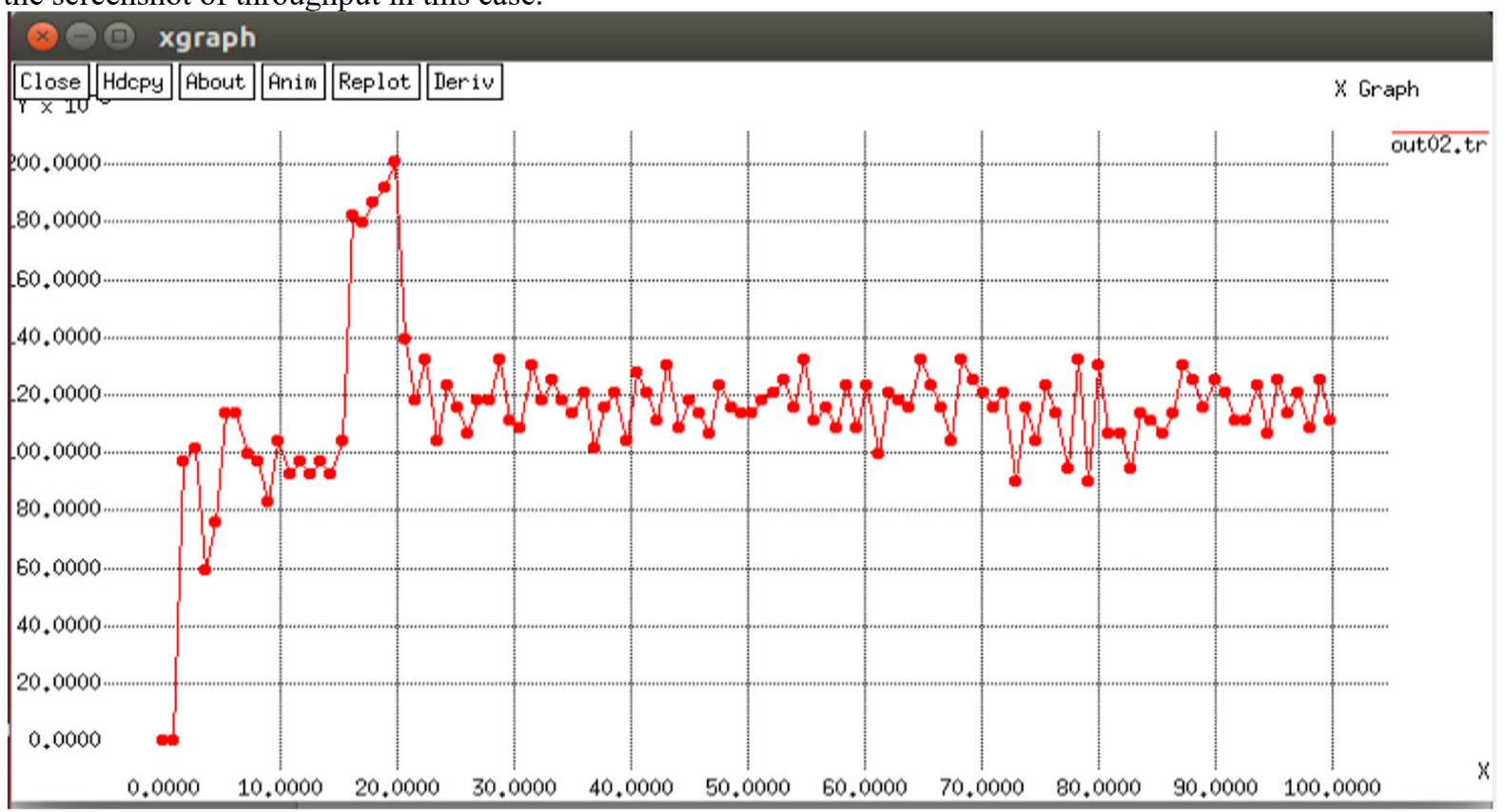

Figure 9. shows the screenshot of throughput in this case 2.

Figure 9 shows that the throughput increases as sink node move to optimum location. This indicate when the distance between sink node and node decreases the throughput increases and delay decreases.

Figure 9 shows the delay in the test case 2 when delay.tr is executed. 


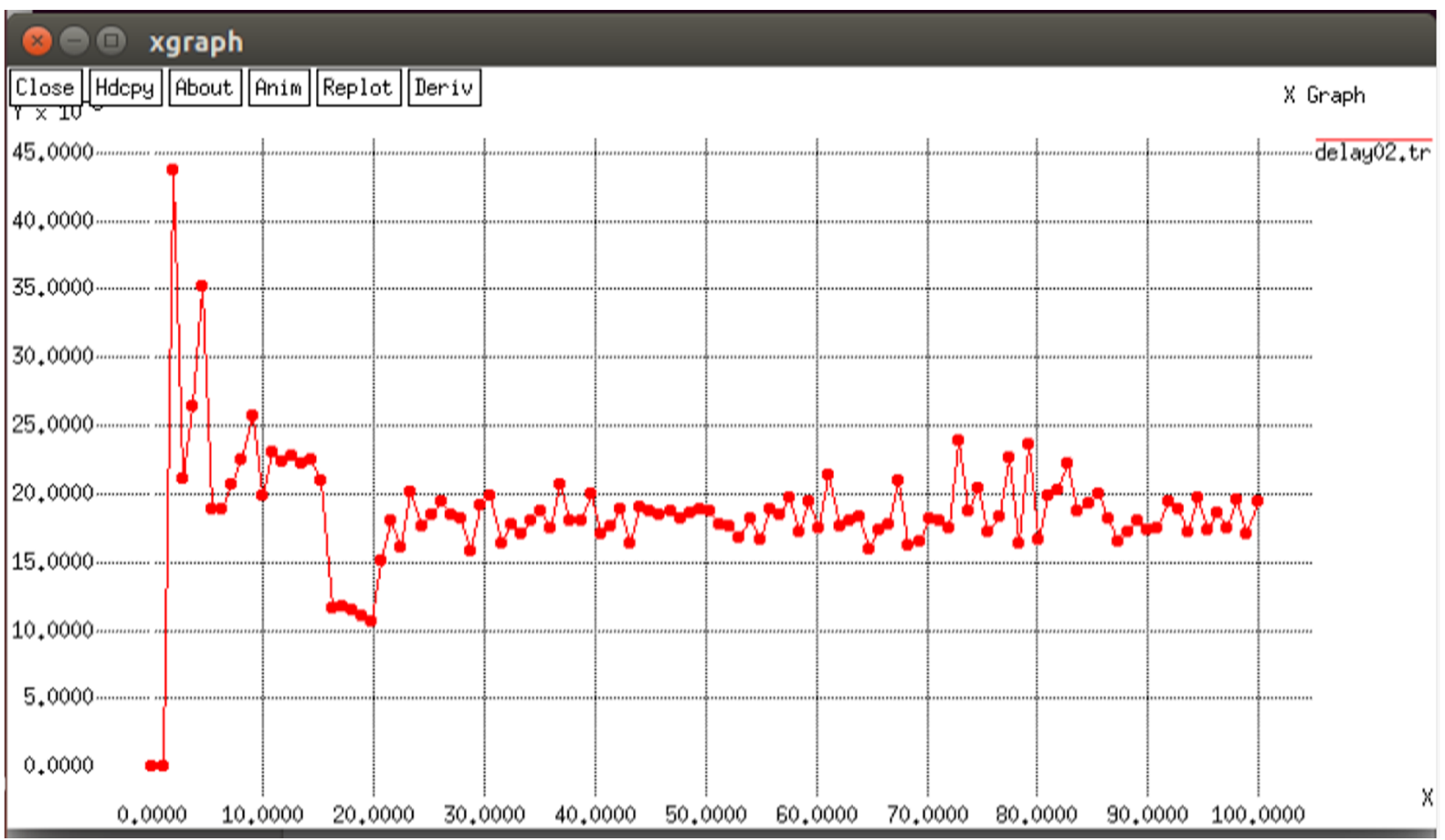

Figure 10. shows the screenshot of throughput in this case 2 .

Figure 10 shows that the delay decreases as sink node move to optimum location. This indicate when the distance between sink node and sensor node decreases the throughput increases and delay decreases.

Test case 2 is tested for AODV output figure 11 number of sent packet, number of received packet, average end-to end delay, number of dropped packet and throughput.

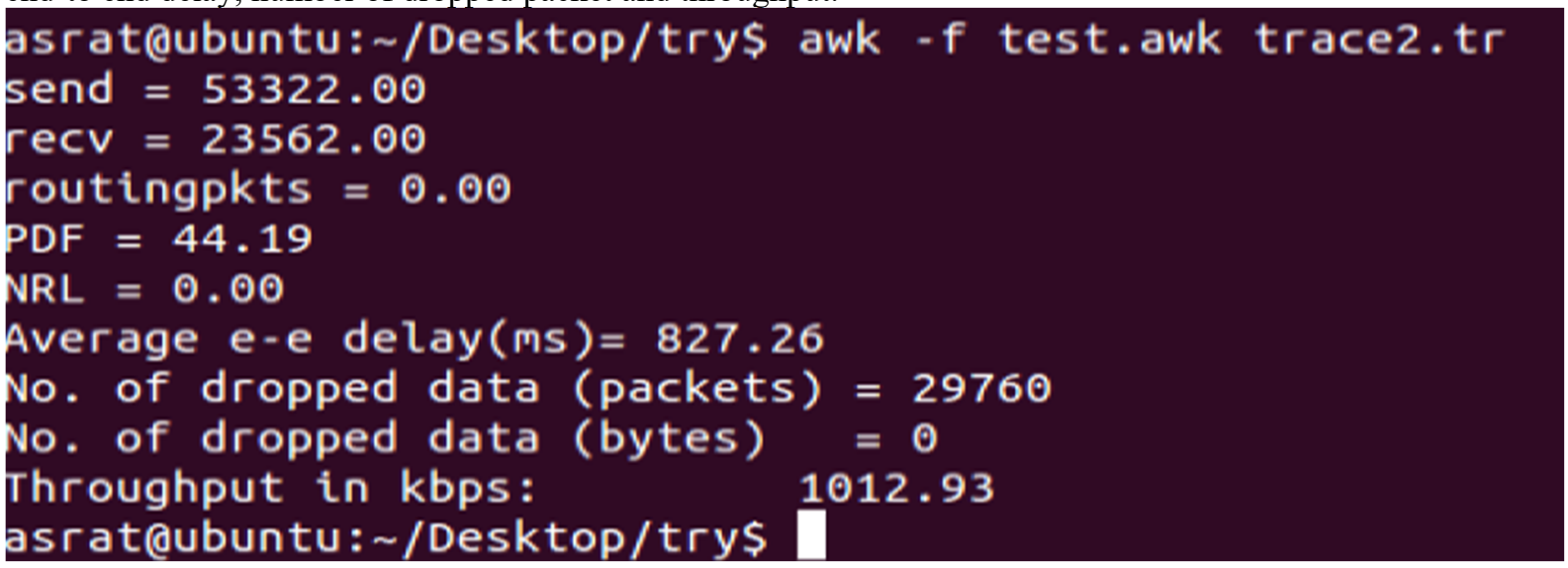

Figure 11. shows the screenshot of packet information in test case 2 .

Average end-to-end delay $=827.26 \mathrm{~ms}$

Throughput $=1012.93 \mathrm{kbps}$

Generally, as we have seen from xgraph and awk file when sink node is at fixed place the throughput decreases and delay increases. But when sink node move to optimum position the throughput increases and delay decreases which increase quality of communication which meet our goal of study.

\section{Conclusion and Future Work}

\subsection{Conclusion}

Generally, this paper evaluates the performances WSN using throughput and delay parameters with Sink repositioning techniques Sink relocation is best approach to decrease distance between sensor node and sink node by repositioning in side region. In this paper we have investigated the performance of sink repositioning in term of throughput and delay. Simulation result shows that such relocation of sink decreases the distance between sink node sensor node which results increase throughput and decrease delay. The implementation of result shown by using NS 2.35 prove that sink repositioning has big deference in term of throughput and delay when sink node at fixed place and after sink node move to optimum place inside region. It achieved significant amount of performance in terms of delay and throughput. The overall performance of sink node relocation was better than 
static sink in term of throughput and delay.

\subsection{Future Works}

As a future work, the authors plan to extend the approach that will allow multiple sinks and create cluster within a region and selecting cluster head and calculate distance of each cluster from sensor node and from cluster head to calculate throughput and delay. Furthermore, we plan to show energy consumption of node during multiple sink movement to optimum location by creating cluster and show the distance and energy consumption at each level.

\section{Conflicts of Interest}

The authors declare no conflicts of interest regarding the publication of this paper.

\section{References}

[1] I. F. Akyildiz, W. Su, Y. Sankarasubramaniam, E. Cayirci, "Wireless sensor networks: survey", Computer Networks, Vol. 38, pp. 393-422, 2002.

[2] C-Y. Chong and S.P. Kumar, "Sensor networks: Evolution, opportunities, and challenges," Proceedings of the IEEE, Vol. 91, No. 8, pp. 1247- 1256, 2003.

[3] Amir Sepasi Zahmati, Bahman Abolhassani, Ali Asghar Beheshti Shirazi, and Ali Shojaee Bakhtiari, "An Energy-Efficient Protocol with Static Clustering for Wireless Sensor Networks", International Journal of Electronics, Circuits and Systems, Vol. 1, No. 2, 2007.

[4] Yang Yu, Viktor K. Prasanna, Bhaskar Krishnamachari, "Information Processing and Routing in Wireless Sensor Networks", World Scientific, Publishing Co. Pte. Ltd. 2006.

[5] Chu-Fu Wang, Jau-Der Shih, Bo-Han Pan, and Tin-Yu Wu "A Network Lifetime Enhancement Method for Sink Relocation and Its Analysis in Wireless Sensor Networks" IEEE SENSORS JOURNAL, VOL. 14, NO. 6, JUNE 2014

[6] GUO, JINGXING, "Sink Mobility Schemes in Wireless Sensor Networks for Network Lifetime Extension" (2012). Electronic Theses and Dissertations. Paper 103.

[7] Mohamed Younis et al, "Base-Station Repositioning For Optimized Performance of Sensor Networks", Vehicular Technology Conference, IEEE 6-9 Oct., 2003.

[8] Mrs. Prerana Shrivastava and Dr. S.B Pokle "Sink Repositioning Technique to Improve the Performance of the Wireless Sensor Networks" International Journal of Future Computer and Communication, Vol. 1, No. 4, December 2012

[9] Kapang Lego, Pranav Kumar Singh, Dipankar Sutradhar, "Comparative Study of Adhoc Routing Protocol AODV, DSR and DSDV in Mobile Adhoc NETwork”, Indian Journal of Computer Science and Engineering, Vol. 1 No. 4 364-371

[10] Gamal, A.El. and Mammen, J. and Prabhakar, B. and Shah, D. "Throughput-delay trade-off in wireless networks, $\|$ in INFOCOM 2004, pp. 464-475

[11] Mrs. Prerana Shrivastava and Dr. S.B Pokle “An Energy Efficient Sink Repositioning Technique for Data Gathering In Wireless Sensor Networks" of the Intl. Conf. on Advances in Computer, Electronics and Electrical Engineering,2012

[12] Gupta, G. and Younis, M. Performance evaluation of loadbalanced clustering of wireless sensor networks. In Telecommunications, 10th International Conference,(2003), 1577 - 158

[13] Asrat Sime Bedada, Prof. Kuda Nageswara Rao" Single Sink Repositioning Technique in Wireless Sensor Networks For Network life Time Extension" International Research Journal of Engineering and Technology (IRJET) e-ISSN: 2395-0056; Volume: 04 Issue: 10 | Oct -2017

[14] Tapan Kumar Jain, Davinder Singh Saini, and Sunil Vidya Bhooshan "Lifetime Optimization of a Multiple Sink Wireless Sensor Network through Energy Balancing" Hindawi Publishing Corporation Journal of Sensors Volume 2015, Article ID 921250,

[15] Wint Yi Poe et al. "Sink Placement without Location Information in Large-Scale Wireless Sensor Networks" AINTEC'09, November 18-20, 2009 